This is the author's final, peer-reviewed manuscript as accepted for publication. The publisher-formatted version may be available through the publisher's web site or your institution's library.

\title{
Affectionate communication received from spouses predicts stress hormone levels in healthy adults
}

Kory Floyd \& Sarah Riforgiate

\section{How to cite this manuscript}

If you make reference to this version of the manuscript, use the following information:

Floyd, K., \& Riforgiate, S. (2008). Affectionate communication received from spouses predicts stress hormone levels in healthy adults. Retrieved from http://krex.ksu.edu

\section{Published Version Information}

Citation: Floyd, K., \& Riforgiate, S. (2008). Affectionate communication received from spouses predicts stress hormone levels in healthy adults. Communication Monographs, 75(4), 351-368.

Copyright: @ 2008 National Communication Association

Digital Object Identifier (DOI): doi:10.1080/03637750802512371

Publisher's Link:

http://www.tandfonline.com/doi/abs/10.1080/03637750802512371\#.UvlUeWJdXL8

This item was retrieved from the K-State Research Exchange (K-REx), the institutional repository of Kansas State University. K-REx is available at http://krex.ksu.edu 
Running head: MARITAL AFFECTION AND STRESS

\author{
Affectionate Communication Received from Spouses Predicts \\ Stress Hormone Levels in Healthy Adults
}

Kory Floyd \& Sarah Riforgiate

Arizona State University

Communication Monographs

Author notes. Kory Floyd is associate professor of human communication at Arizona State University, where Sarah Riforgiate is a doctoral student. This study was funded by a grant to the senior author from the Office of the Vice Provost for Research at Arizona State University. Address correspondence to the senior author at Hugh Downs School of Human Communication, Arizona State University, PO Box 871205, Tempe AZ 85287-1205. (480) 965-3568. Electronic mail: kory@asu.edu 


\begin{abstract}
Recent research on the communication of affection has illuminated its implications for mental and physical health. In particular, affectionate communication has been shown to covary with healthy hormonal variation and accelerated recovery from stress. The present study focuses on the association between marital affection and hormonal markers of stress regulation, including cortisol, dehydroepiandrosterone-sulfate (DHEA-S), and their ratio. Twenty healthy adults and their spouses provided independent reports of their propensity for verbal, nonverbal, and supportbased expressions of affection prior to providing saliva samples that were assayed for cortisol and DHEA-S. As hypothesized, spouses' reports of verbal, nonverbal, and supportive affection significantly predicted participants’ waking cortisol levels, cortisol change, and cortisol:DHEA-S ratio. Participants' own reports of affection were predictive of cortisol:DHEA-S ratio for verbal affection behaviors only, and were not predictive of participants’ waking cortisol, cortisol change, or DHEA-S. In addition, spouses’ reports of verbal, nonverbal, and supportive affection predicted participants’ evening cortisol levels. Results illustrate that affectionate communication from one's spouse is related to hormonal stress regulation and suggest the possibility that interventions designed to increase affectionate behavior in romantic relationships may have stress-ameliorating physiological effects.
\end{abstract}




\section{Affectionate Communication Received from Spouses Predicts \\ Stress Hormone Levels in Healthy Adults}

The expression of affection is among the most consequential communicative behaviors for the formation and maintenance of marriages and other significant pair bonds (Huston, Caughlin, Houts, Smith, \& George, 2001; King \& Christensen, 1983). One reason why sharing affectionate communication may benefit marital partners is that it may help to diffuse the negative mental and physical effects of stress (see Floyd, 2006a). Several recent investigations, detailed below, have demonstrated that affection exchange in personal relationships is associated with healthy stress regulation, which benefits individuals and may also benefit their relationships by extension. The present study tests the hypothesis that spouses' levels of affectionate communication with each other predict hormonal markers of stress regulation, including waking levels of the adrenal hormone cortisol, magnitude of morning-to-evening (diurnal) change in cortisol, levels of the adrenal prohormone dehydroepiandrosterone-sulfate (DHEA-S), and the cortisol:DHEA-S ratio (abbreviated hereafter as CDR). As detailed below, stress is inversely related to affectionate communication, whereas it is directly related to CDR and inversely related to DHEA-S, waking cortisol, and diurnal variation in cortisol. We expected, therefore, that marital affection predicts waking cortisol, cortisol variation, DHEA-S, and CDR, such that higher levels of affectionate behavior are associated with higher DHEA-S, waking cortisol, and cortisol variation levels and lower CDR scores. For exploratory purposes, we also examined associations with cortisol levels measured at other points during the day. 
Subsequently, we describe the relationship between affectionate communication and stress, and then provide detail regarding the biochemical markers of stress investigated herein. Specific hypotheses and research questions are then advanced.

\section{Affectionate Communication and Stress}

The notion that affectionate behavior might ameliorate stress can trace its roots at least back to Harry Harlow's pioneering research in the mid-20th Century. Before Harlow’s work, experts discouraged overt expressions of affection between parents and children. Psychologists predicted that receiving affectionate behavior would make children needy and demanding, and physicians warned that it would promote the spread of infectious diseases (Blum, 2002). Harlow's now-classic experiments with infant macque monkeys suggested, however, that the need for affection is pervasive (even to the point of overriding more fundamental needs such as the need for food) and that receiving even inauthentic "expressions” of affection (via tactile contact with terrycloth-covered wire figures) induced calm and mitigated the effects of stressors (Harlow, 1958).

In the last half century, various theories in the social sciences have taken up the question of why, and in what ways, affectionate behavior between humans might have some of the same stress-ameliorating psychological and physiological effects that Harlow observed among infant macques. For instance, tend and befriend theory (TBT: Taylor, Klein, Lewis, Gruenewald, Gurung, \& Updegraff, 2000) provides that behavior aimed at fortifying personal relationships (such as the expression of affection) is beneficial for health because it ameliorates the physiological stress response. In particular, TBT implicates the pituitary hormone oxytocin in the stress-reducing effects of tending and befriending behaviors. Similarly, the stress-buffering hypothesis (SBH: Cohen, Doyle, Skoner, \& Gwaltney, 1997;) suggests that the more social 
support people receive from their personal relationships, the less susceptible they are to the negative effects of stressors. Both TBT and SBH therefore imply that communicative behaviors aimed at building and maintaining significant, intimate, and supportive social relationships such as the communication of affection - should be inversely associated with susceptibility to stress. This is efficacious to health not only in its own right, but also in light of evidence that exaggerated cardiovascular and hormonal responses to stressors are associated with elevated risk of cardiovascular disease (Lynch, Everson, Kaplan, Salonen, \& Salonen, 1998), as well as hypertension and associated organ damage (Manuck, Kasprowicz, \& Muldoon, 1990).

TBT and SBH both share conceptual space with affection exchange theory (AET: Floyd, 2002, 2006a), a neo-Darwinian theory providing that the tendency for humans to communicate affection is an adaptive mechanism for promoting the development of pair bonds. Among its principal proposals is that conveying affection has stress-ameliorating physiological effects. Specifically, AET provides that expressing affection to loved ones initiates neuroendocrine processes that maximize reward and buffer the individual against the physiological effects of stress; AET further provides that these benefits are independent of those associated with receiving affectionate expressions.

Multiple studies have illustrated this effect. For instance, Floyd (2006b) examined the effects of expressed and received affection on diurnal variation in cortisol and found that, with the influence of received affection controlled for, expressed affection was directly related to the magnitude of morning-to-evening change in cortisol $(\beta=.56)$, a pattern indicative of healthy stress regulation in the hypothalamic-pituitary-adrenal axis. A subsequent experiment by Floyd, Mikkelson et al. (in press) showed that during episodes of acute stress (in which cortisol levels are typically elevated), expressing affection to a loved one accelerates the recovery of cortisol 
levels to baseline values, relative to alternative activities. Grewen, Girdler, Amico, and Light (2005) also found that engaging in nonverbal affectionate behaviors reduced cortisol levels for both men and women, and also elevated levels of oxytocin in women (see also Turner, Altemus, Enos, Cooper, \& McGuinness, 1999). Floyd, Mikkelson, Hesse, and Pauley (2007) demonstrated that expressing affection in writing significantly lowers total serum cholesterol (which is elevated by stress). Finally, Floyd, Hesse, and Haynes (2007) reported a strong inverse association ( $\beta=-.85$ ) between expressed affection and glycohemoglobin, an index of average blood glucose level (which is also elevated by stress), with the effects of received affection controlled for.

Hormonal Stress Markers

From a physiological standpoint, the experience of stress is perhaps best understood as the body's regulatory response to environmental threats, which include any perceived challenge to one's physical, mental, emotional, financial, or social well-being. Perception of a stressor initiates a cascade of physiological changes associated with the fight-or-flight response; among these is a multistage hormonal response regulated by the hypothalamic-pituitary-adrenal (HPA) axis. Two results of this cascade are an elevated level of cortisol and a reduced level of DHEAS, as described below. Importantly, even events that fail to elevate psychological distress can initiate a physiological stress response. For instance, one commonly used laboratory stressor, the cold pressor test (e.g., Riozzi, Heagerty, Bing, Thurston, \& Swales, 1987), requires participants to hold a forearm submerged in ice water. Although most participants report that this is not a psychologically stressful experience, the typical participant experiences an immediate and pronounced physiological stress response. In this section, we provide detail on cortisol and DHEA-S, and also on the cortisol:DHEA-S ratio, which are measured in this study. 
Cortisol. Cortisol is a steroid hormone secreted by the adrenal gland. In response to acute stressors, the HPA axis elevates the secretion of cortisol into the bloodstream. Approximately 95\% of the secreted cortisol binds immediately to corticosteroid binding globulins and albumin, rendering it biologically inactive. The remainder, known as free cortisol, remains biologically active in order to mobilize the body's energy resources to mount an adequate defense against the stressor. This involves various physiological effects, including an elevation in blood glucose, a diversion of energies from nonessential bodily systems, and an increase in immune sensitivity (Johnson, Karmilaris, Chrousos, \& Gold, 1992; Sapolsky, 2002). Absent acute stressors, cortisol follows a diurnal rhythm wherein it peaks in the hour after waking and drops continually during the day, reaching its lowest point around midnight (Kirschbaum \& Hellhammer, 1989). Highly differentiated diurnal curves are indicative of healthy regulation of the body's stress defenses, whereas "flattened" curves reflect dysregulation (Giese, Sephton, Abercrombie, Duran, \& Spiegel, 2004).

Dehydroepiandrosterone-sulfate. Dehydroepiandrosterone-sulfate (DHEA-S), a derivative of dehydroepiandrosterone, is also an adrenal steroid. Unlike cortisol, DHEA-S is not a hormone itself, but rather an important prohormone, meaning that it is a substance that converts to other hormones (Labrie et al., 2005). Specifically, DHEA-S is a precursor to androstenedione, testosterone, and estrogens in adolescents and adults (Labrie, Luu-The, Labrie, \& Simard, 2001). DHEA-S is more frequently studied in social science research because it has a longer half-life than DHEA and an absence of diurnal variation (Rosenfeld, Rosenberg, Fukushima, \& Hellman, 1975). DHEA and DHEA-S appear to have protective functions against a variety of conditions, including HIV (Loria, Inge, Cook, Szakal, \& Regelson, 1988), Cushing’s disease (Yamaji, Ishibashi, Sekihara, Itabashi, \& Yanaihara, 1984), Alzheimer’s disease 
(Näsman et al., 1995), and some forms of cancer (Gordon, Helzlsouer, \& Comstock, 1991). Importantly, whereas cortisol is elevated in response to stress, DHEA-S is decreased. In a laboratory procedure involving experimentally induced acute stress, for instance, Whetzel, Ritter, and Klein (2006) found that participants experienced a 40\% decrease in DHEA-S levels, on average, after just one 20-minute mental math (serial subtraction) stressor.

Cortisol:DHEA-S ratio. Previous research has suggested that the ratio of cortisol to DHEA-S may be as reliable an indicator of stress, if not more so, than the absolute levels of either chemical (see, e.g., Cruess et al., 1999). When mathematically expressed as cortisol divided by DHEA-S (rather than the obverse), CDR should show direct associations with stress, since stress increases levels of the numerator (cortisol) and decreases levels of the denominator (DHEA-S) (see Parker, Levin, \& Lifrak, 1985). Previous investigations have demonstrated that CDR is directly associated with multiple diverse forms of stress, including surgical stress (Ozasa, Kita, Inoue, \& Mori, 1990), bodily stress due to anorexia nervosa (Zumoff et al., 1983) or injury (Butcher, Killampalli, Lascelles, Wang, Alpar, \& Lord, 2005), laboratory induced stress (Whetzel et al., 2006) or fear (Grillon, Pine, Baas, Lawley, Ellis, \& Charney, 2005), and the stress of military school (Morgan et al., 2004), and can be significantly decreased by a cognitivebehavioral stress management intervention (Cruess et al., 1999). Hypotheses and Research Question

To the extent that higher levels of affectionate communication are predictive of lower levels of stress (as AET provides and as previous investigations, reported above, have shown), we can advance predictions about its association with hormonal stress markers based on the physiology of those markers. Specific hypotheses are delineated subsequently. 
Cortisol. Since circulating cortisol levels increase in response to acute stress, it may seem logical to predict that chronic stress is associated with elevated mean cortisol levels. In fact, however, stress is indicated by the magnitude of morning-to-evening variation in cortisol, rather than by its mean level. As discussed above, healthy regulation of the HPA axis is indicated by cortisol levels that are high upon awakening and drop sharply over the course of the day, whereas dysregulation is indicated by low waking levels and minimal diurnal variation. Multiple studies have confirmed that chronic stress is inversely related to waking cortisol level (Leucken, Dausch, Gulla, Hong, \& Compas, 2004; Neylan et al., 2005; Yang et al., 2001) and to the magnitude of diurnal variation in cortisol (Chrousos \& Gold, 1992; Heim, Ehlert, \& Hellhammer, 2000). When considered in concert with AET’s prediction that affectionate communication ameliorates stress, these observations give rise to two specific predictions:

H1: In marital relationships, affectionate communication predicts waking cortisol level, such that higher amounts of affection correspond to higher waking values.

H2: In marital relationships, affectionate communication predicts diurnal variation in cortisol, such that higher amounts of affection correspond to higher morning-toevening change in cortisol levels.

Although waking cortisol is the most directly implicated in stress, cortisol values at other points during the day may also be affected by spouses' affectionate communication. We addressed this possibility with a research question:

RQ1:How, if at all, does marital affectionate communication predict cortisol levels at noon, afternoon, and evening? 
DHEA-S. As indicated above, DHEA-S manifests an inverse relationship with stress. To the extent that affectionate communication is similarly inversely related to stress, it is logical to hypothesize a direct relationship between affectionate communication and DHEA-S level:

H3: In marital relationships, affectionate communication predicts mean dehydroepiandrosterone-sulfate, such that higher amounts of affection correspond to higher DHEA-S levels.

Cortisol:DHEA-S ratio. CDR is elevated by stress, as detailed above. To the extent to that affectionate communication ameliorates stress, it is therefore logical to hypothesize an inverse predictive association between affectionate expression in the marital relationship and CDR:

H4: In marital relationships, affectionate communication predicts cortisol:DHEA-S ratio, such that higher amounts of affection correspond to lower ratio values.

Method

\section{Participants}

Participants $(n=20)$ and their legal spouses $(n=20)$ were equal numbers of adult men and women composing 20 married couples. Participants and spouses both ranged in age from 22 to 57 years, with average ages of 40.50 years $(S D=13.59)$ for participants and 40.10 years $(S D=$ 14.12) for spouses. The couples had been married an average of 13 years, 4 months ( $S D=$ 135.81 months) and had an average of 2.5 children $(S D=4.73)$. The majority ( $65 \%$ of participants, $90 \%$ of spouses) was Caucasian; $10 \%$ of participants and spouses were Hispanic, $10 \%$ of participants were Asian, $5 \%$ of participants were Native American, and $10 \%$ of participants claimed other ethnic origins. Half of the participants and spouses had at least a 4- 
year college degree, with $25 \%$ of participants and $20 \%$ of spouses holding a graduate degree. A majority (60\%) came from households earning more than $\$ 60,000$ per year.

Recruitment and Prescreening

Undergraduate communication students at a large university in the southwestern United States were given extra course credit for recruiting qualified married couples to complete prescreening questionnaires. To qualify, prospective participants had to be English-speaking adults (18+ years of age) who had been legally married for at least one year, and both the husband and wife had to agree to fill out a prescreening questionnaire asking about their medicinal use and history of illness. The prescreening questionnaires were accompanied by postage-paid envelopes, which prospective participants used to return them to the researchers. Each was surveyed upon receipt to determine the eligibility of prospective participants.

Stringent inclusion and exclusion criteria were imposed. Prospective participants were considered eligible to take part in the study only if they: a) were normotensive; b) reported no history of immune or endocrine disease, cancer of any form, kidney or liver disease, cardiological or respiratory problems, rheumatological disorders, or diabetes; c) had never had chemotherapy or chest radiation; d) were not currently taking steroidal medications, alpha or beta blockers, or oral contraceptives; e) were non-smokers; f) were not currently pregnant; g) were not currently breastfeeding; and, h) reported consuming no more than three caffeinated drinks per day and three alcoholic beverages per week, on average.

One hundred forty three couples completed the prescreening form. Among these, $34 \%$ of men and $41 \%$ of women met all of the qualifications for the study. This sex difference was statistically significant, $z=2.20, p<.05$. From among these, 20 randomly selected couples, half including an eligible husband and half including an eligible wife, were contacted and invited to 
take part in the study. Three initially invited couples declined to participate; these were replaced with randomly selected alternates.

Procedure

Eligible couples who agreed to take part were sent by overnight delivery a package containing two questionnaires with postage-paid return envelopes, one kit for collecting saliva samples, and detailed instructions. Participants and spouses independently completed questionnaires regarding their individual and relational communication patterns and mailed them separately to the researchers.

In each couple, either the husband or the wife (whomever was assigned to be the participant) was then instructed to take four saliva samples over the course of a normal workday (as defined by the participant). The samples were taken by unstimulated passive drool in marked plastic cryovials at awakening, noon, 4 p.m., and bedtime. Participants were instructed to abstain from caffeine on the collection day and not to consume any food or beverage other than water for at least one hour prior to each collection. Participants provided self-reports of their compliance with these instructions on a form submitted to the laboratory with their samples. Noncompliance with the instructions would not necessarily invalidate a given sample; rather, it would simply increase measurement error (see, e.g., Wolkowitz \& Rothschild, 2003). After completing their salivary collections, participants sealed their collection wells first into a plastic biohazard bag and then into a marked cardboard box, and sent them via FedEx Clinical Pak to BioHealth Diagnostics, a service laboratory in San Diego, CA. Clinical investigations have verified that salivary levels correlate closely with free plasma levels for both cortisol (Tunn, Möllmann, Barth, Derendorf, \& Krieg, 1992) and DHEA-S (Lac, Lac, \& Robert, 1993). 
In consideration of their participation, each couple was subsequently sent \$20 and an informational booklet about stress and adrenal health. The spouse providing the saliva samples was also sent a copy of his or her assay results. The research protocol was approved by the university’s institutional review board.

Questionnaire Measure

Affectionate communication was assessed with the factor-based Affectionate Communication Index (ACI: Floyd \& Morman, 1998). The 18-item Likert-type scale indexes affection expressed through verbal behaviors (such as saying "I love you”), direct nonverbal behaviors (such as kissing or hugging), and social support behaviors (such as praising accomplishments or helping with problems). The ACI has evidenced multiple forms of psychometric adequacy, including high internal, split-half, and test-retest reliability, and construct, convergent, discriminant, and predictive validity (for review, see Floyd \& Mikkelson, 2005). In the present study, Cronbach’s alpha values for participants and spouses, respectively, were .84 and .92 for verbal affection, .92 and .88 for nonverbal affection, and .75 and .74 for supportive affection.

\section{Biochemical Measures}

Hormonal assays were performed by BioHealth Diagnostics Laboratory (San Diego, CA). Upon arrival, the saliva samples were frozen pending analysis. On the day of the assays, the samples were thawed and centrifuged at 3000 rotations per minute for 15 minutes, and then assayed for cortisol and DHEA-S, as well as for other biochemical markers not reported here. BioHealth Diagnostics is a CLIA-certified laboratory, meaning that it maintains standards of quality as specified by the Clinical Laboratory Improvement Amendments enacted by Congress in 1988 (license 05D0982456). 
Cortisol. The saliva samples were assayed for free cortisol, expressed in nanomoles per liter (nMol/L), using a commercially available, highly sensitive enzyme immunoassay cleared for use as an in vitro diagnostic measure of adrenal function (Salimetrics, State College, PA). In the assay, salivary cortisol competes with cortisol linked to horseradish peroxidase for the antibody binding sites. After incubation, the amount of cortisol peroxidase detected is inversely proportional to the amount of salivary cortisol present. The test is sensitive enough to detect cortisol levels as small as $<0.003$ micrograms per deciliter $(\mu \mathrm{g} / \mathrm{dL})$ and has an average intraassay coefficient of variation of $3.50 \%$ and average inter-assay coefficient of variation of 5.08\% (both of which are measures of reliability wherein lower percentages represent greater reliability).

DHEA-S. Samples were assayed for average DHEA-S, expressed in nanograms per milliliter (ng/mL), using a commercially available competitive enzyme immunoassay (Salimetrics, State College, PA). The assay uses a microtitre plate coated with rabbit antibodies to DHEA-S. Salivary estradiol competes with DHEA-S linked to horseradish peroxidase for the antibody binding sites. After incubation, the amount of DHEA-S peroxidase detected is inversely proportional to the amount of salivary DHEA-S present. The test can detect DHEA-S levels as small as 5 picograms per milliliter $(\mathrm{pg} / \mathrm{mL})$ and has an average intra-assay coefficient of variation of $5.60 \%$ and average inter-assay coefficient of variation of $8.20 \%$.

Results

Descriptive Statistics

Affectionate communication. Descriptive statistics for participants' and spouses’ reports of verbal, nonverbal, and supportive affectionate communication appear in Table 1. A mixedmodel MANOVA indicated no significant differences by sex or role (participant vs. spouse) on 
any of the forms of affectionate communication (all $p s>.05$ ). Participants' verbal affection was inversely related to participants' age, $r(18)=-.50, p=.026$. Spouses’ verbal affection was likewise inversely related to spouses' age, $r(18)=-.57, p=.008$. Age was unrelated to nonverbal and supportive forms of affectionate behavior for both participants and spouses.

Biochemical measures. Cortisol routinely shows strong diurnal variation, typically peaking within the hour after awakening and then dropping continuously during the day, reaching its lowest level around midnight (Kirchbaum \& Hellhammer, 1989). By contrast, DHEA-S does not evidence diurnal variation, with levels remaining stable throughout the day (Diagnostic Systems Laboratory, 1995). To establish the CDR while accounting for diurnal variation in cortisol, therefore, we calculated the ratio based on cortisol values summed across the four data collection periods. Ratio values represent the sum of cortisol values divided by DHEA-S. To index cortisol change over the day, we computed linear orthogonal polynomials using a formula espoused by Pennebaker, Mayne, and Francis (1997) (who computed similar polynomials to index change in the linguistic features of expressive writing). The specific formula was:

(evening cortisol x 3) + (afternoon cortisol x 1) - (noon cortisol x 1) - (waking cortisol x 3) Descriptive statistics for DHEA-S, CDR, cortisol change, and average cortisol values at awakening, noon, afternoon, and evening appear in Table 2. Women and men differed significantly on waking cortisol, with women $(M=21.39 \mathrm{nMol} / \mathrm{L}, S D=5.24)$ evidencing higher levels than men $(M=16.19, S D=4.40), t(18)=-2.40, p=.027, d=-1.13$. Women also had higher evening cortisol values $(M=2.58 \mathrm{nMol} / \mathrm{L}, S D=1.17)$ than did men $(M=1.53, S D=$ 0.98), $t(18)=-2.18, p=.043, d=-1$.03. Finally, women evidenced greater morning-to-evening cortisol change $(M=-58.57, S D=14.81)$ than did men $(M=-45.88, S D=11.61), t(18)=2.13, p$ 
$=.047, d=1.00$. There were no significant sex differences on the other cortisol values, DHEAS, or CDR. Participant and spouse ages showed no significant zero-order correlations with any of the biochemical outcomes.

Hypotheses and Research Question

The hypotheses predicted that affectionate communication within marital relationships predicts participants' waking cortisol (H1), cortisol change (H2), DHEA-S (H3), and CDR (H4), such that higher levels of affectionate communication correspond to higher waking cortisol, greater cortisol change, higher DHEA-S, and lower CDR. The research question asked whether cortisol values at noon, afternoon, and evening are predicted by marital affection. To address the hypotheses and research question in tandem, we computed hierarchical linear regressions with the three forms of affectionate communication as criterion variables.

Because we had reports of marital affection both from participants and from their spouses, we tested both. We entered participant sex, participant age, and spouse age in the first step of each regression as control variables, as these have the potential to influence the biochemical outcomes (see, e.g., Laughlin \& Barrett-Connor, 2000). Initially, we also entered as a control variable the partner's value for the specific form of affectionate behavior being tested (e.g., spouse's verbal affection was a control variable when participant's verbal affection was the predictor), but these control variables were nonsignificant in every regression and were therefore removed in the service of parsimony.

Centered values for participants’ and spouses’ reports of verbal, nonverbal, and supportive affection were then entered as separate predictors in the second step of each regression. Square-root transformed values for waking cortisol, cortisol change, DHEA-S, and CDR were each entered as the criterion variable. (In the case of CDR, all control variables were 
nonsignificant, so we removed them from the regressions, resulting in bivariate regressions with affectionate communication as the sole predictor variable.) Although the biochemical variables were square-root transformed in the regression analyses, we report raw (non-transformed) values in the tables for ease of interpretation.

Waking cortisol (H1). When we analyzed participants’ reports of how much affection they express to their spouses, we found that neither their verbal, nonverbal, nor supportive forms of affectionate communication predicted their waking cortisol values. When we analyzed spouses' reports of how much affection they express to the participants, however, we found that participants' waking cortisol was significantly predicted by spouses’ verbal affection, $\beta=.59, p$ $=.003$; nonverbal affection, $\beta=.45, p=.022$; and supportive affection, $\beta=.55, p=.007$. The hypothesis is fully supported with respect to how much affection participants receive from their spouses, but not supported with respect to how much affection participants express to their spouses. Regression results appear in Table 3.

Other cortisol values (RQ1). Participants’ and spouses’ reports of verbal, nonverbal, and supportive affection were all unrelated to participants' cortisol levels at noon and in the afternoon. Participants’ reports were likewise unrelated to their evening cortisol levels. However, participants’ evening cortisol values were significantly predicted by their spouses’ verbal affection, $\beta=.59, p=.01$; nonverbal affection, $\beta=.56, p=.01$; and supportive affection, $\beta=.58, p=.01$. Regression results appear in Table 4 .

Cortisol change (H2). Cortisol change was calculated using linear orthogonal polynomials such that all values representing morning-to-evening reduction are negative, and greater morning-to-evening change is represented by scores with higher absolute value (i.e., -.74 indexes greater change than -.21). Therefore, the hypothesis that affectionate communication 
predicts cortisol change, such that higher amounts of affection correspond to higher amounts of cortisol change, would be supported by beta scores that are negative, not positive. Participants' cortisol change was not predicted by their own verbal, nonverbal, or supportive affection. It was, however, significantly predicted by their spouses' verbal affection, $\beta=-.55, p=.006$; nonverbal affection, $\beta=-.40, p=.04$; and supportive affection, $\beta=-.51, p=.01$. Regression results appear in Table 5. The second hypothesis is supported with respect to spouses' affectionate communication.

DHEA-S (H3). Participants' mean DHEA-S levels were not predicted by their own verbal, nonverbal, or supportive affection, nor by their spouses' verbal, nonverbal, or supportive affection. The third hypothesis is unsupported.

Cortisol:DHEA-S ratio (H4). When we analyzed participants' reports of how much affection they express to their spouses (using bivariate regressions, as indicated above), we found that only their verbal affection significantly predicted their CDR, $\beta=-.51, p=.01$. The associations were inverse but nonsignificant for participants’ nonverbal and supportive affection. When we analyzed spouses' reports of how much affection they express to the participants, however, we found that participants’ CDR was significantly predicted by spouses’ verbal affection, $\beta=-.39, p=.045$; nonverbal affection, $\beta=-.42, p=.03$; and supportive affection, $\beta=$ $-.41, p=.04$. The hypothesis is fully supported with respect to how much affection participants receive from their spouses, and partially supported with respect to how much affection participants express to their spouses.

\section{Discussion}

Recent research has illuminated the inverse association between affectionate communication and the physiological characteristics of stress, including diurnal cortisol variation 
(Floyd, 2006b), cortisol reactivity (Floyd et al., in press), blood glucose (Floyd, Hesse et al., 2007), and serum cholesterol (Floyd, Mikkelson et al., 2007). The current study extended this line of research in two important ways: first, it examined the relative influence of affection expressed to, and affection received from, a significant relational partner; second, it investigated the predictive associations between affectionate communication and multiple physiological stress markers in tandem.

Among the innovative proposals of affection exchange theory is that affectionate communication elicits stress-ameliorating effects both when it is expressed and when it is received, and previous studies have confirmed that expressed and received affection account for unique (as well as shared) variance in outcomes related to physical and mental well-being (Floyd, Hess, Miczo, Halone, Mikkelson, \& Tusing 2005), including assessments of stress and depression. On this basis, we hypothesized that affectionate communication expressed from participants to their spouses, and affectionate communication received by participants from their spouses, would both predict the participants' biochemical stress parameters. Although earlier investigations had identified associations between affectionate behavior and cortisol, this study was the first also to examine DHEA-S and the cortisol:DHEA-S ratio, each of which is strongly implicated in the management of stress.

Affectionate communication received from spouses was consistently more strongly related to healthy stress hormone levels than was participants' affection expressed to their spouses. Specifically, participants' waking cortisol levels, evening cortisol levels, diurnal cortisol change, and CDR were all significantly predicted by how much verbal, nonverbal, and supportive affection they receive from their spouses (even when controlling for participants' expressed affection). These findings add to a growing body of evidence that affectionate 
communication in personal relationships contributes to the efficient and effective management of physiological stress.

This observation is provocative, given the number and variety of physical conditions that are exacerbated by stress, including coronary artery disease (Blascovich, Shiffert, \& Katlin, 1989), hypertension (Potempa, 1994), elevated cholesterol and cardiovascular disease (Roy, Kirschbaum, \& Steptoe, 2001), and immunosuppression (Kiecolt-Glaser, Garner, Speicher, Penn, Holliday, \& Glaser, 1984; Kiecolt-Glaser, Glaser, Shuttleworth, Dyer, Ogrocki, \& Speicher, 1987). Since an individual's hormonal stress parameters are predicted by the amount of affectionate communication he or she receives from a spouse, this fuels the possibility that behavioral interventions designed to increase affectionate communication with the marital relationship may improve these parameters (e.g., increasing 24-hour cortisol variance, reducing CDR values). Indeed, some affection-oriented interventions have already shown efficacy in preliminary trials. In two five-week randomized controlled trials, for instance, Floyd, Mikkelson et al. (2007) demonstrated that an affectionate writing intervention significantly lowered total serum cholesterol, and current research in our lab is testing the efficacy of nonverbal affection interventions for improving immunocompetence. These efforts are useful because stress is such a pervasive public health threat; indeed, McEwan (1999) estimated the economic cost of stress and stress-related illness in the United States alone at nearly \$200 billion annually. Even interventions that manifest statistically small effects may therefore represent clinically significant improvements in health and quality of life.

The sample size of the present study was small relative to that typically seen in interpersonal communication research; however, it was well within the norm for psychophysiological studies (e.g., Kurup \& Kurup, 2003; Marazziti \& Canale, 2004; van 
Niekerk, Huppert, \& Herbert, 2001), including psychophysiological studies conducted within the field of interpersonal communication (e.g., Floyd, 2006b; Tardy, Thompson, \& Allen, 1989). The emergence of several significant predictive associations in the current study and the relative immunity of biochemical measures from the influence of social desirability biases both argue for the adequacy of the sample, although confidence in external validity would be enhanced with the inclusion of more couples.

As described, participants were extensively prescreened and were subjected to numerous inclusion and exclusion criteria related to their health-related practices, medicinal use, and history of illnesses and therapies. These criteria were necessary to ensure the efficacy of the salivary analyses; however, they resulted in a sample that was no doubt healthier, on average, than the population from which it was drawn. This is a common conundrum in research using psychophysiological methods; whereas the sample must have specific characteristics to make the physiological measurements valid, those characteristics often reduce the sample’s external validity. Replication of the procedures with a non-screened sample will not necessarily improve confidence in external validity, therefore, since violation of the inclusion and exclusion criteria may make the physiological measures inaccurate.

These limitations aside, the present findings point clearly to a predictive association between affectionate communication received from a spouse and the management of one’s own hormonal stress markers. This association appears to be relatively independent of the type of affectionate communication being expressed (predictive relationships having attained statistical significance for all three forms of affectionate communication, in most cases). It is possible that this predictive association reflects a causal relationship wherein patterns of affection exchange determine, or partially determine, stress hormone levels. It is also possible that both affection 
exchange patterns and stress hormone levels are influenced by exogenous factors such as external sources of relational stress. Such factors might mediate the association between affectionate communication and stress hormone levels, or they might render the association spurious. Affection exchange theory would support the hypothesis that patterns of affection exchange exert genuine causal influences on stress hormone levels but would not necessarily rule out the possibility that such influences are mediated by exogenous factors. Identifying the extent to which the affection-hormone association is directly causal, mediated, or the spurious result of a third variable will require additional experimental work, but these significant predictive associations provide ample warrant for such studies. 


\section{References}

Blascovich, J., Shiffert, J. H., \& Katkin, E. H. (1989). A comparison of exercise and psychophysiological stress testing for coronary disease. Psychophysiology, 26, 57.

Blum, D. (2002). Love at goon park: Harry Harlow and the science of affection. Cambridge, MA: Perseus.

Butcher, S. K., Killampalli, V., Lascelles, D., Wang, K., Alpar, E. K., \& Lord, J. M. (2005). Raised cortisol:DHEAS ratio in the elderly after injury: Potential impact of neurophil function and immunity. Aging Cell, 4, 391-324.

Chrousos, G., \& Gold, P. (1992). The concepts of stress and stress system disorders. Journal of the American Medical Association, 267, 1244-1252.

Cohen, S., Doyle, W. J., Skoner, D. P., Rabin, B. S., \& Gwaltney, J. M. (1997). Social ties and susceptibility to the common cold. Journal of the American Medical Association, 277, 1940-1944.

Cruess, D. G., Antoni, H. M., Kumar, M., Ironson, G., McCabe, P., Fernandez, J. B., Fletcher, M., \& Schneiderman, N. (1999). Cognitive-behavioral stress management buffers descreases in dehydroepiandrosterone sulfate (DHEA-S) and increases in the cortisol/DHEA-S ratio and reduces mood disturbance and perceived stress among HIVseropositive men. Psychoneuroendocrinology, 24, 537-549.

Diagnostic Systems Laboratory. (1995). Active DHEA-S Kit (DSL-3500).

Floyd, K. (2006a). Communicating affection: Interpersonal behavior and social context. Cambridge, England: Cambridge University Press. 
Floyd, K. (2006b). Human affection exchange: XII. Affectionate communication is associated with diurnal variation in salivary free cortisol. Western Journal of Communication, 70, 4763.

Floyd, K., Hess, J. A., Miczo, L. A., Halone, K. K., Mikkelson, A. C., \& Tusing, K. J. (2005). Human affection exchange: VIII. Further evidence of the benefits of expressed affection. Communication Quarterly, 53, 285-303.

Floyd, K., Hesse, C., \& Haynes, M. T. (2007). Human affection exchange: XV. Metabolic and cardiovascular correlates of trait expressed affection. Communication Quarterly, 55, 79-94.

Floyd, K., Mikkelson, A. C., Hesse, C., \& Pauley, P. M. (2007). Affectionate writing reduces total cholesterol: Two randomized, controlled trials. Human Communication Research, 33, 119-142.

Floyd, K., Mikkelson, A. C., Tafoya, M. A., Farinelli, L., La Valley, A. G., Judd, J., Haynes, M. T., Davis, K. L., \& Wilson, J. (in press). Human affection exchange: XIII. Affectionate communication accelerates neuroendocrine stress recovery. Health Communication.

Gordon, G. B., Helzlsouer, K. J., \& Comstock, G. W. (1991). Serum levels of dehydroepiandrosterone and its sulfate and the risk of developing bladder cancer. Cancer Research, 51, 1366-1369.

Grewen, K. M., Girdler, S. S., Amico, J., \& Light, K. C. (2005). Effects of partner support on resting oxytocin, cortisol, norepinephrine, and blood pressure before and after warm partner contact. Psychosomatic Medicine, 67, 531-538.

Grillon, C., Pine, D. S., Baas, J. M. P., Lawley, M., Ellis, V., \& Charney, D. S. (2005). Cortisol and DHEA-S are associated with startle potentiation during aversive conditioning in humans. Psychopharmacology, 186, 434-441. 
Harlow, H. F. (1958). The nature of love. American Psychologist, 13, 573-685.

Heim, C., Ehlert, U., \& Hellhammer, D. H. (2000). The potential role of hypocorticolism in the pathophysiology of stress-related bodily disorders. Psychoneuroendocrinology, 25, 1-35.

Huston, T. L., Caughlin, J. P., Houts, R. M., Smith, S. E., \& George L. J. (2001). The connubial crucible: Newlywed years as predictors of marital delight, distress, and divorce. Journal of Personality and Social Psychology, 80, 237-252.

Kiecolt-Glaser, J. K., Garner, W., Speicher, C., Penn, G. M., Holliday, J., \& Glaser, R. (1984). Psychosocial modifiers of immunocompetence in medical students. Psychosomatic Medicine, 46, 7-14.

Kiecolt-Glaser, J. K., Glaser, R., Shuttleworth, E. C., Dyer, C. S., Ogrocki, P., \& Speicher, C. E. (1987). Chronic stress and immunity in family caregivers of Alzheimer's disease victims. Psychosomatic Medicine, 49, 523-535.

King, C. E., \& Christensen, A. (1983). The relationship events scale: A Guttman scaling of progress in courtship. Journal of Marriage and the Family, 45, 671-678.

Kirschbaum, C., \& Hellhammer, D. H. (1989). Salivary cortisol in psychobiological research: An overview. Neuropsychology, 22, 150-169.

Kurup, R. K., \& Kurup, P. A. (2003). Hypothalamic digoxin, hemispheric dominance, and neurobiology of love and affection. International Journal of Neuroscience, 113, 721729.

Labrie, F., Luu-The, V., Labrie, C., \& Simard, J. (2001). DHEA and its transformation into androgens and estrogens in peripheral target tissues: Intracrinology. Frontiers in Neuroendocrinology, 22, 185-212. 
Labrie, F., Luu-The, V., Bélanger, A., Lin, S.-X., Simard, J., Pelletier, G., \& Labie, C. (2005). Is dehydroepiandrosterone a hormone? Journal of Endocrinology, 187, 169-196.

Lac, G., Lac, N., \& Robert, A. (1993). Steroid assays in saliva: A method to detect plasmatic contaminations. Archives Internationales de Physiologie et de Biochimie, 101, 257-262.

Laughlin, G. A., \& Barrett-Connor, E. (2000). Sexual dimorphism in the influence of advanced aging on adrenal hormone levels: The Rancho Bernardo study. Journal of Clinical Endocrinology and Metabolism, 85, 3561-3568.

Loria, R. M., Inge, T. H., Cook, S. S., Szakal, A. K., \& Regelson, W. (1988). Protection against acute lethal viral infections with the native steroid dehydroepiandrosterone (DHEA). Journal of Medical Virology, 26, 301-314.

Luecken, L. J., Dausch, B., Gulla, V., Hong, R., \& Compas, B. E. (2004). Alterations in morning cortisol associated with PTSD in women with breast cancer. Journal of Psychosomatic Research, 56, 13-15.

Lynch, J. W., Everson, S. A., Kaplan, G. A., Salonen, R., \& Salonen, J. T. (1998). Does low socioeconomic status potentiate the effects of heightened cardiovascular responses to stress on the progression of carotid atherosclerosis? American Journal of Public Health, 88, 389-394.

Manuck, S. B., Kasprowicz, A. L., \& Muldoon, M. F. (1990). Behaviorally evoked cardiovascular reactivity and hypertension: Conceptual issues and potential associations. Annals of Behavioral Medicine, 12, 17-29.

Marazziti, D., \& Canale, D. (2004). Hormonal changes when falling in love. Psychoneuroendocrinology, 29, 931-936. 
McEwan, B. (1999). Stress and the brain. In R. Conlan (Ed.), States of mind: New discoveries about how our brains make us who we are (pp. 81-102). New York: Wiley.

Morgan, C. A., Southwick, S., Hazlett, G., Rasmusson, A., Hoyt, G., Zimolo, Z., \& Charney, D. (2004). Relationships among plasma dehydroepiandrosterone sulfate and cortisol levels, symptoms of dissociation, and objective performance in humans exposed to acute stress. Archives of General Psychiatry, 61, 819-825.

Näsman, B., Olsson, T., Seckl, J. R., Eriksson, S., Viitanen, M., Bucht, G., \& Carlstrom, K. (1995). Abnormalties in adrenal androgens, but not of glucocorticoids, in early Alzheimer's disease. Psychoneuroendocrinology, 20, 83-94.

Neylan, T. C., Brunet, A., Pole, N., Best, S. R., Metzler, T. J., Yehuda, R., \& Marmar, C. R. (2005). PTSD symptoms predict waking cortisol levels in police officers. Psychoneuroendocrinology, 30, 373-381.

Ozasa, H., Kita, M., Inoue, T., \& Mori, T. (1990). Plasma dehydroepiandrosterone to cortisol ratios as an indicator of stress in gynecological patients. Gynecological Oncology, 37, 178-182.

Parker, L. N., Levin, E. R., \& Lifrak, E. T. (1985). Evidence for adrenocortical adaptation to severe illness. Journal of Clinical Endocrinology and Metabolism, 60, 947-952.

Pennebaker, J. W., Mayne, T. J., \& Francis, M. E. (1997). Linguistic predictors of adaptive bereavement. Journal of Personality and Social Psychology, 72, 863-871.

Potempa, K. (1994). An overview of the role of cardiovascular reactivity to stressful challenges in the etiology of hypertension. Journal of Cardiovascular Nursing, 8, 27-38. 
Riozzi, A., Heagerty, A. M., Bing, R. F., Thurston, H., \& Swales, J. D. (1987). Effects of cold pressor test-induced stress on leukocyte sodium transport and norepinephrine. Hypertension, 9, 13-17.

Rosenfeld, R. S., Rosenberg, B. J., Fukushima, D. K., \& Hellman, L. (1975). 24-hour secretory pattern of dehydroepiandrosterone and dehydroepiandrosterone sulfate. Journal of Clinical Endocrinology and Metabolism, 40, 850-855.

Roy, M. P., Kirschbaum, C., \& Steptoe, A. (2001). Psychological, cardiovascular, and metabolic correlates of individual differences in cortisol stress recovery in young men. Psychoneuroendocrinology, 26, 375-391.

Tardy, C. H., Thompson, W. R., \& Allen, M. T. (1989). Cardiovascular responses during speech: Does social support mediate the effects of talking on blood pressure? Journal of Language and Social Psychology, 8, 271-285.

Taylor, S. E., Klein, L. C., Lewis, B. P., Gruenewald, T. L., Guring, R. A. R., \& Updegraff, J. A. (2000). Biobehavioral responses to stress in females: Tend-and-befriend, not fight-orflight. Psychological Review, 107, 411-429.

Tunn, S., Möllmann, H., Barth, J., Derendorf, H., \& Krieg, M. (1992). Simultaneous measurement of cortisol in serum and saliva after different forms of cortisol administration. Clinical Chemistry, 38, 1491-1494.

Turner, R. A., Altemus, M., Enos, T., Cooper, B., \& McGuinness, T. (1999). Preliminary research on plasma oxytocin in normal cycling women: Investigating emotion and interpersonal distress. Psychiatry, 62, 97-113. 
van Niekerk, J. K., Huppert, F. A., \& Herbert, J. (2001). Salivary cortisol and DHEA: Association with measures of cognition and well-being in normal older men, and effects of three months of DHEA supplementation. Psychoneuroendocrinology, 26, 591-612.

Whetzel, C. A., Ritter, F. E., \& Klein, L. C. (2006). DHEA-S and cortisol responses to stress and caffeine in healthy young men: Is DHEA-S a reliable marker for stress? Psychosomatic Medicine, 68, A-77.

Wolkowitz, O. M., \& Rothschild, A. J. (Eds.). (2003). Psychoneuroendocrinology: The scientific basis of clinical practice. Arlington, VA: American Psychiatric Publishing, Inc.

Yamaji, T., Ishibashi, M., Sekihara, H., Itabashi, A., \& Yanaihara, T. (1984). Serum dehydroepiandrosterone sulfate in Cushing's syndrome. Journal of Clinical Endocrinology and Metabolism, 59, 1164-1168.

Yang, Y., Koh, D., Ng, V., Lee, F. C., Chan, G., Dong, F., \& Chia, S. F. (2001). Salivary cortisol levels and work-related stress among emergency department nurses. Journal of Occupational and Environmental Medicine, 43, 11-18.

Zumoff, B., Walsh, B. T., Katz, J. L., Levin, J., Rosenfeld, R. S., Kream, J., \& Weiner, H. (1983). Subnormal plasma dehydroepiandrosterone to cortisol ratio in anorexia nervosa: A second hormonal parameter of ontogenic regression. Journal of Clinical Endocrinology and Metabolism, 56, 668-672. 
Table 1

Descriptive Statistics for Participants' and Spouses' Affectionate Communication $(N=40)$

\begin{tabular}{lcccccc}
\hline & \multicolumn{2}{c}{ Participants' Reports } & \multicolumn{3}{c}{ Spouses’ Reports } \\
Form of Affection & Low/High & $M$ & $S D$ & Low/High & $M$ & $S D$ \\
& & & & & & \\
Verbal & $2.80 / 7.00$ & 5.25 & 1.13 & $1.60 / 7.00$ & 4.79 & 1.70 \\
Nonverbal & $2.25 / 7.00$ & 5.26 & 1.31 & $2.00 / 7.00$ & 4.82 & 1.30 \\
Support-Based & $4.20 / 7.00$ & 5.87 & 0.73 & $4.20 / 7.00$ & 5.82 & 0.86 \\
& & & & & & \\
\hline
\end{tabular}

Notes. All variables were measured on 1-7 scales wherein higher numbers indicate greater values. None of the variables differed significantly by sex or by role (participant vs. spouse), all ps $>.05$. 
Table 2

Descriptive Statistics and Intercorrelations for Participants’ Biochemical Markers $(N=20)$

\begin{tabular}{|c|c|c|c|c|c|c|c|c|c|c|}
\hline Marker & Low & High & $M$ & $S D$ & 2 & 3 & 4 & 5 & 6 & 7 \\
\hline 1. Cortisol (waking) & 10.3 & 26.8 & 18.79 & 5.41 & .57 & .28 & .69 & .64 & .09 & -.98 \\
\hline 2. Cortisol (noon) & 4.4 & 11.3 & 7.82 & 1.71 & & .84 & .27 & .36 & .21 & -.59 \\
\hline 3. Cortisol (afternoon) & 2.4 & 9.2 & 5.80 & 1.80 & & & .04 & .19 & .19 & -.28 \\
\hline 4. Cortisol (evening) & 0.6 & 4.1 & 2.06 & 1.18 & & & & .54 & -.03 & -.56 \\
\hline 5. DHEA-S & 1.73 & 6.28 & 3.02 & 1.06 & & & & & -.64 & -.60 \\
\hline 6. Cortisol:DHEA-S ratio & 6.6 & 17.5 & 12.01 & 2.69 & & & & & & -.11 \\
\hline 7. Cortisol change & -29.20 & -75.30 & -52.23 & 14.50 & & & & & & \\
\hline
\end{tabular}

Notes. Cortisol values are expressed in nMol/L. DHEA-S values are expressed in ng/mL. Cortisol:DHEA-S ratio represents cortisol sum divided by DHEA-S level. Cortisol change is indexed by linear orthogonal polynomials. 
Table 3

Regressions Predicting Participants’ Waking Cortisol from Spouses’ Verbal, Nonverbal, and Supportive Affectionate Communication

Predictor

Zero-order $r$

$B$

SE B

$\beta$

Step 1: Participant sex

.49

6.08

2.01

$.58 * *$

Participant age

.13

1.19

0.45

2.98*

Spouse age

.06

$-1.18$

0.43

$-3.08 *$

Step 2: Spouse verbal

.30

1.88

0.54

$.59 * *$

$R^{2}=.72 ;$ adjusted $R^{2}=.64 ; F(4,15)=9.41, p=.001$

Step 2: Spouse nonverbal

1.87

0.73

$.45^{*}$

$R^{2}=.64 ;$ adjusted $R^{2}=.55 ; F(4,15)=6.72, p=.003$

Step 2: Spouse supportive

.05

3.48

1.11

$.55^{* *}$

$R^{2}=.69 ;$ adjusted $R^{2}=.61 ; F(4,15)=8.29, p=.001$

Notes. ${ }^{*} p<.05 ; * * p<.01$ 
Table 4

Regressions Predicting Participants' Evening Cortisol from Spouses’ Verbal, Nonverbal, and Supportive Affectionate Communication

Predictor

Zero-order $r$

$B$

SE B

$\beta$

Step 1: Participant sex

.46

1.34

0.50

$.59 *$

Participant age

$-.17$

0.04

0.11

.47

Spouse age

$-.17$

$-0.07$

0.11

$-.84$

Step 2: Spouse verbal

.48

0.41

0.15

$.59 *$

$R^{2}=.56 ;$ adjusted $R^{2}=.45 ; F(4,15)=4.84, p=.01$

Step 2: Spouse nonverbal

.42

0.51

0.17

$.56^{*}$

$R^{2}=.58 ;$ adjusted $R^{2}=.47 ; F(4,15)=5.14, p=.008$

Step 2: Spouse supportive

.26

0.80

0.29

$.58^{*}$

$R^{2}=.56 ;$ adjusted $R^{2}=.44 ; F(4,15)=5.76, p=.011$

Notes. ${ }^{*} p<.05 ; * * p<.01$ 
Table 5

Regressions Predicting Participants' Cortisol Change from Spouses' Verbal, Nonverbal, and Supportive Affectionate Communication

Predictor

Zero-order $r$

$B$

SE B

$\beta$

Step 1: Participant sex

$-.45$

$-14.54$

5.34

$-.51 *$

Participant age

$-.18$

$-3.59$

1.18

$-3.36 * *$

Spouse age

$-.10$

3.48

1.14

$3.39 * *$

Step 2: Spouse verbal

$-.25$

$-4.73$

1.49

$-.55 * *$

$R^{2}=.70 ;$ adjusted $R^{2}=.62 ; F(4,15)=8.66, p=.001$

Step 2: Spouse nonverbal

$-.11$

$-4.45$

2.02

$-.40 *$

$R^{2}=.62 ;$ adjusted $R^{2}=.52 ; F(4,15)=6.11, p=.004$

Step 2: Spouse supportive

$-.01$

$-8.57$

3.08

$-.51 *$

$R^{2}=.67 ;$ adjusted $R^{2}=.58 ; F(4,15)=7.54, p=.002$

Notes. ${ }^{*} p<.05 ;{ }^{* *} p<.01$ 